\title{
Subcellular Proteomics and Global Analysis of Posttranslational Modifica- tions to Study Functional Roles of Trypanosoma cruzi Molecules
}

\author{
Igor C. Almeida* and Ernesto S. Nakayasu*, ,l
}

The Border Biomedical Research Center, Department of Biological Sciences, University of Texas at El Paso, El Paso TX, 79968, USA

\begin{abstract}
One century after the discovery of Chagas disease, the treatment for this illness is still based only on two drugs with limited efficacy and severe side effects. In this mini-review, we discuss the application of mass spectrometry (MS)based proteomic approaches to study the biochemistry and cell biology of etiologic agent of Chagas disease, Trypanosoma cruzi. We focus the discussion in the analysis of subcellular proteomics and posttranslational modifications (PTMs). In recent years, subcellular proteomics has brought new insights into the localization of proteins and possible functions of organelles. Thus far, proteomic analysis of reservosomes, ribosomes, detergent-solubilized membranes, and a preparation of an organelle mixture have been performed. In addition, a number of analyses of PTMs of $T$. cruzi proteins (i.e., histone modifications, phosphorylation, glycosylation, glycosylphosphatidylinositol (GPI)-anchoring, and nitrosylation) have been successfully carried out. The identification of those and other PTMs combined with cutting-edge biochemical, immunological and cell biology approaches, have allowed a more in-depth understanding of biological and pathophysiological processes resulting from host cell-parasite interactions.
\end{abstract}

Keywords: Trypanosoma cruzi, Chagas disease, proteomics, posttranslational modifications, mass spectrometry.

\section{INTRODUCTION}

Chagas disease or American trypanosomiasis was discovered one century ago by Carlos Chagas. Chagas disease is a major public health concern in Latin America, where about 11 million people suffer of this illness and other 120 million people live in risk areas [1-4]. Of those infected, about 50 thousand may die every year due to complications in the acute and chronic phases of the disease. Chagas disease is also becoming an important public health issue in the USA and Europe due to migration of chronically infected, asymptomatic people from endemic areas [1-4]. This situation is aggravated due to the dearth of regular screening for this disease in blood banks and hospitals [5-7].

Trypanosoma cruzi is the etiologic agent of Chagas disease and has four different life-cycle stages or forms, two in the insect-vector and two in the mammalian host (Fig. 1A). In the reduviid insect, popularly known as kissing bug, epimastigotes proliferate in the midgut [8]. Under nutritional stress, the epimastigote forms migrate to the distal region of the gut and convert into nonproliferative infective forms called metacyclic trypomastigotes, which are then released with the excreta during the insect bloodmeal (Fig. 1A). Metacyclic trypomastigotes are able to enter the host bloodstream through the bite wound or exposed mucosal tissues, and infect a variety of nucleated cells. Inside host cells they differentiate into amastigote forms, which reproduce by

*Address correspondence to these authors at the Border Biomedical Research Center, Department of Biological Sciences, University of Texas at El Paso, 500 West University Avenue, El Paso, TX 79968, USA; Tel: +1 (915) 747-6898/+1 (915) 747-6086; Fax: +1 (915) 747-5808;

E-mail: icalmeida@utep.edu, esnakayasu@miners.utep.edu

${ }^{\text {IC }}$ Current address: Pacific Northwest National Laboratory, P.O. Box 999, Richland, WA 99352, USA. binary fission and transform into infective trypomastigotes (Fig. 1A). These forms are then released from the cells into the extracellular milieu to infect surrounding cells, further reaching the bloodstream to infect remote tissues or a new kissing bug, thus completing the natural life cycle (Fig. 1A) [8].

Besides the natural route of transmission, T. cruzi can be transmitted by transplantation, blood transfusion, congenitally, and by contaminated foods and fluids $[3,4,9]$. Current therapy is based only on two drugs, namely benznidazole and nifurtimox. Both of these compounds have limited efficacy and may cause severe side effects [10]. Thus, there is an urgent need for developing new, more effective chemotherapies. Furthermore, although many efforts have been made in the past years, there is no available vaccine for treating or preventing Chagas disease [11-13]. We hypothesize that one of the hurdles in developing an effective vaccine is that most if not all experimental vaccines have been based on a single protein or peptide(s) derived thereof. Frequently, there is little or no evidence that the target antigen is homogenously expressed by infective bloodstream trypomastigotes from different strains and lineages that could infect humans.

T. cruzi genome is composed by several multigene families, such as trans-sialidase (TS)/gp85 glycoproteins, mucins, mucin-associated surface proteins (MASP), retrotransposon hot spot (RHS), and gp63. Each of these families includes several hundreds of genes, which could be concurrently expressed $[14,15]$. For developing effective immunotherapies, it is crucial to know exactly which and when these genes are expressed. To aggravate this already complex scenario, $T$. cruzi has three major lineages ( $T$. cruzi I, II, and III) $[16,17]$, which were recently further reclassified into six discrete typing units (DTUs) (i.e., T. cruzi I-VI) [18], each 
one of them encompassing numerous strains expressing different subsets of genes and having distinct biological and pathophysiological traits $[17,18]$. Moreover, gene expression in $T$. cruzi is polycistronic and the levels of transcript usually do not correlate with the amount of protein being expressed [19]. Thus, approaches such as microarray and cDNA library to map expressed proteins are not consistent. In this context, proteomic analysis is the method of choice to more reliably determine gene expression in $T$. cruzi.

The two mostly used approaches to perform proteomic analysis or proteomics are peptide-mass fingerprint (PMF) and liquid chromatography-tandem mass spectrometry (LCMS/MS) [20-22] (Fig. 1B). In PMF experiments, protein mixtures are separated either by one- (1-DE) or twodimensional gel electrophoresis (2-DE), the bands/spots are excised, digested with specific endoproteinase (usually trypsin), and the resulting peptides analyzed by matrix-assisted laser desorption/ionization-mass spectrometry (MALDI-MS) to determine their accurate molecular masses [20-22]. The peptide identification is achieved by searching a database of known protein sequences, which have been "virtually" (or in silico) digested with same protease used experimentally. Then, the molecular masses of experimental peptides are compared with the theoretical peptide masses, and the results statistically analyzed to define the more reliable matches (Fig. 1B) [20-22]. In LC-MS/MS experiments, a complex mixture of proteins is digested in-solution with a specific protease (usually trypsin) and the resulting peptides are loaded into a reverse phase (RP) capillary column connected to a nanoflow high-performance liquid chromatographer
(nanoHPLC or nanoLC). The eluting peptides are analyzed on-line by electrospray ionization-tandem mass spectrometry (ESI-MS/MS), which not only records the molecular masses of intact peptides, but also individually select and fragment them, generating therefore internal amino acid sequences. The identification is done first by searching the database for the peptide(s) with same molecular mass. To confirm this identification, candidate peptide sequences are submitted to a theoretical fragmentation and compared to the experimental fragmentation (MS/MS) spectrum (Fig. 1B) [20-22]. Of course, depending on the complexity of the sample to be analyzed, a combination of chromatographic techniques can be used for better resolving peptides prior to the LC-MS/MS analysis [23]. For instance, peptides derived from very complex protein mixtures can be fractionated (off-line or on-line) by strong cation-exchange (SCX), RP, strong-anion exchange (SAX) or hydrophilic interaction (HILIC) chromatography prior to the LC-MS/MS analysis [23]. Alternatively, posttranslationally modified (i.e., phosphorylated, glycosylated, etc.) peptides can be enriched using immobilized antibodies or other types of affinity chromatography, such as lectin and immobilized metal-affinity chromatography [2427].

In recent years, many efforts have been concentrated in the proteomic studies of whole cell lysates for the analysis of different $T$. cruzi developmental stages [28-32], cell differentiation process [33], and drug resistance [34]. Expression proteomics of total $T$. cruzi lysate has been extensively discussed in a book chapter that is freely available at NCBI bookshelf webpage (http://www.ncbi.nlm.nih.gov/bookshelf/
A

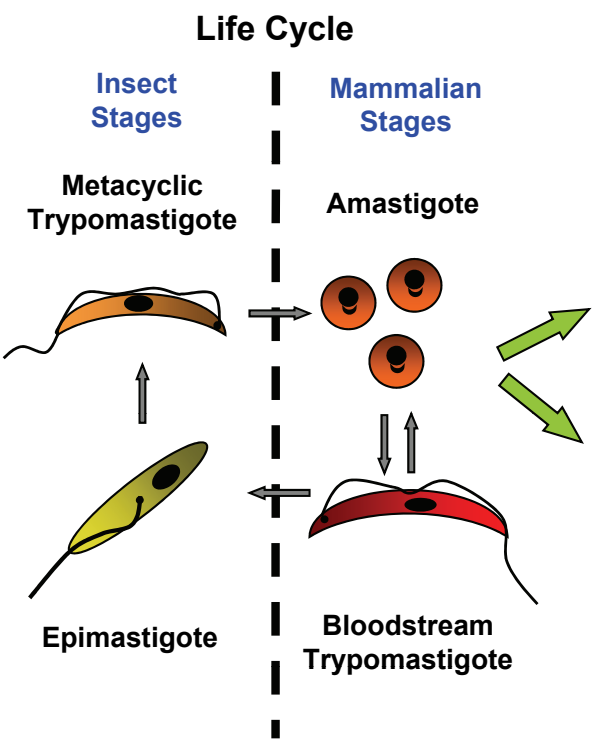

B

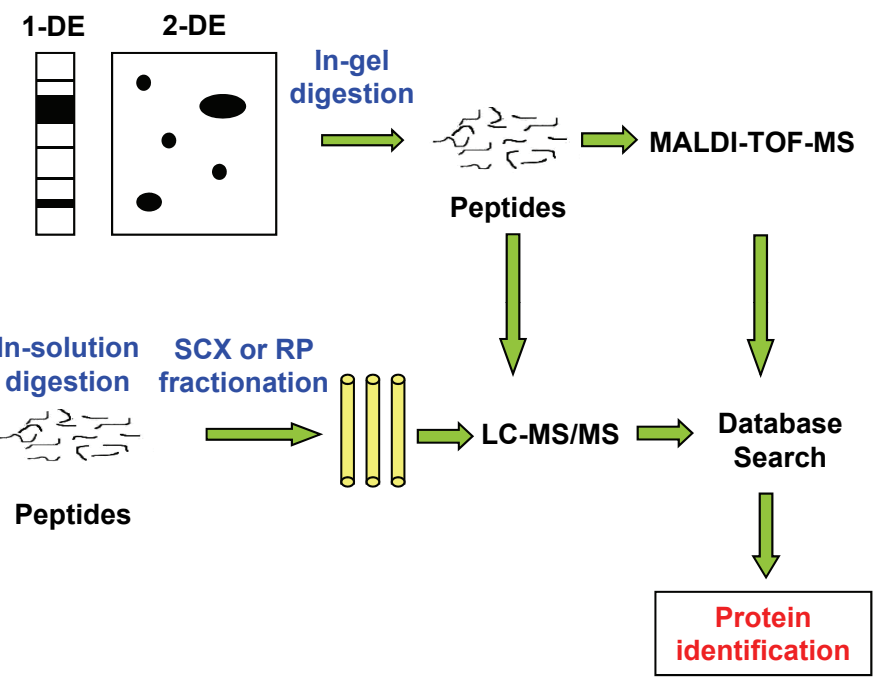

Fig. (1). Trypanosoma cruzi life cycle (A) and general proteomic approaches (B). (A) T. cruzi has two stages in the insect vector, epimastigotes and metacyclic trypomastigotes, and 2 stages in the mammalian host, amastigotes and bloodstream trypomastigotes. (B) Cell lysates of various stages of $T$. cruzi are analyzed by peptide-mass fingerprint (PMF) or liquid chromatography-tandem mass spectrometry (LCMS/MS). In PMF, proteins resolved by 1-DE or 2-DE, are submitted to in-gel digestion and analyzed by matrix assisted laser desorption/ionization-mass spectrometry (MALDI-MS). In another approach, proteins are digested in solution, and the resulting peptides are fractionated using reverse phase (RP) or strong cation-exchange (SCX) chromatography and analyzed by LC-MS/MS. The identification of the proteins is achieved by searching the spectra against a protein sequence database. 
br.fcgi?book=bioinfo\&part=B05). Thus, in this mini-review we will focus the discussion on proteomics of subcellular components and posttranslational modifications (PTMs).

\section{SUBCELLULAR PROTEOMICS}

T. cruzi has many subcellular compartments/organelles with specialized functions, which are not present in higher eukaryotes. Among these organelles/compartments are the kinetoplast (a region inside the parasite sole large mitochondrion, containing the mitochondrial genome), reservosomes (a lysosomal-like organelle exclusively present in epimastigote stage), glycosomes (a peroxisome-like organelle containing several glycolytic enzymes), and alcidocalcisomes (organelles involved in the storage of calcium and phosphorus) $[35,36]$. Other organelles also present in higher eukaryotes may be very divergent, thus having different functions. In this context, the study of subcellular proteomics is particularly interesting and could provide key information about the localization of many proteins and imply biological functions and pathways for many parasite organelles.

Recently, Ferella et al. [37] obtained a subcellular fraction of epimastigotes enriched mainly with acidocalcisomes and glycosomes by ultracentrifugation using an OptiPrep gradient. Proteins from this fraction were separated by 1-DE and 2-DE, digested with trypsin, and analyzed by LCMS/MS (Fig. 2A). This led to the identification of 396 proteins, of which $35 \%$ had previously been annotated as hypothetical proteins. Interestingly, one hundred and seventythree proteins had not previously been identified in the largescale analysis of epimastigote proteome [28]. This improvement in protein coverage might be due to the elimination of highly abundant cytoplasmic proteins that interfere in the proteomic analysis. Despite of the unquestionable contribution of the publication by Ferella et al. [37], the lack of a better purified preparation precluded the assignment of proteins into a single organelle. This makes the identification of new consensus sorting signals that direct proteins into organelles much more difficult. A prediction analysis using previously known targeting signals revealed proteins from many different organelles in the preparation, including mitochondrion, nucleus, flagellum, acidocalcisomes, glycosomes, and plasma membrane. Several proteins were also predicted to have multiple locations. The authors also validated the localization of 5 hypothetical proteins into endoplasmic reticulum (ER), mitochondrion, acidocalcisome, and an unidentified vesicle [37].

\section{Reservosomal Proteomics}

Reservosomes are lysosome-related, nutrient storage organelles which are the final destination of endocytosed proteins and lipids in epimastigote forms of $T$. cruzi [38, 39]. Although reservosomes are not present in other T. cruzi stages (i.e., metacyclic trypomastigotes, amastigotes, and trypomastigotes), these have similar lysosome-related organelles but lacking the ability of storing nutrients [39, 40]. For the proteomic analysis, reservosomes were obtained by lysing the cells with sonication, and fractionating the organelles by ultracentrifugation in a sucrose gradient [41]. A reservosomal membrane fraction was also obtained by disrupting the organelles by sequential freezing and thawing cycles, and treating them with sodium carbonate to remove periph- eral membrane proteins. The purity of the organelle and membrane fractions was assessed by transmission electron microscopy (TEM). Proteins were then digested with trypsin alone or in combination with endoproteinase Glu-C, fractionated by strong cation-exchange (SCX) chromatography, and analyzed by LC-MS/MS (Fig. 2B) [41]. A total of $709 \mathrm{~T}$. cruzi-specific proteins were identified, being $36 \%$ of them annotated as hypothetical proteins. In agreement with the idea of reservomes being a storage organelle, additional 160 proteins were identified mainly from the cell culture medium. Interestingly, many proteins related to the function of reservosomes such as hydrolases, endosomal/lysosomal proteins, proteins involved in vesicular traffic, and pump and channel proteins, were also found [41]. Since reservosome can be a recycling organelle, proteins from other compartments could also be there to be degraded. To confirm the reservosome preparation was free of significant contamination with other organelles, the reservosomal proteome was compared to subcellular proteomes of glycosomes, mitochondrion, and flagellum of $T$. brucei [41-44]. It was clearly shown that most of the highly abundant proteins from those preparations were not present in the enriched reservosomal fraction, suggesting that the impurities were negligible [41].

\section{Ribosomal Proteomics}

T. cruzi has major differences in transcription mechanism as compared to other eukaryotes. First, the genome is organized in large polycistronic clusters, which are transcribed en bloc and processed into individual mRNAs. As a result of this processing each mRNA receives at the 5'-end a 39nucleotide miniexon or spliced leader (SL) $[45,46]$. Since $T$. cruzi has unusual mRNAs, the structure of the ribosomal complex could also be different from other eukaryotes. The ribosome and its subunits are classified by the coefficient of sedimentation, being the complete complex called $80 \mathrm{~S}$, and the major subunits $60 \mathrm{~S}$ and $40 \mathrm{~S}$ [47]. T. cruzi $80 \mathrm{~S}$ ribosomes purified by differential centrifugation and ultracentrifugation in sucrose gradient and analyzed by cryo-electron microscopy (cryo-EM) showed some differences that could be associated with the binding of the SL [47, 48]. Purified ribosomes were then digested with trypsin and analyzed by $2 \mathrm{D}$ LC-MS/MS (Fig. 2C). Thirty-two proteins from the 40S ribosomal subunit were found, whereas 47 were identified in the $60 \mathrm{~S}$ subunit [48]. Several proteins were shown to have extensions or deletions in either $\mathrm{N}$ - or C-terminus, which could explain the differences observed in the cryo-EM experiments. Nevertheless, it remains to be determined whether these differences are responsible for the binding of the SL.

\section{Membrane Proteomics}

Membrane proteins such as channels, transporters, receptors, enzymes, and ligands play key roles in many cellular processes. In parasites, surface membrane proteins are of great interest since they are in direct contact with host cells and the extracellular environment [15, 49]. Furthermore, these proteins are frequently implicated in the ability of the parasite to invade host cells and escape the host immune response; therefore, they have been exploited as potential targets for vaccine development [11-13]. Membrane proteins are challenging to be analyzed since they are poorly soluble in aqueous buffers $[50,51]$. In order to compare membrane 


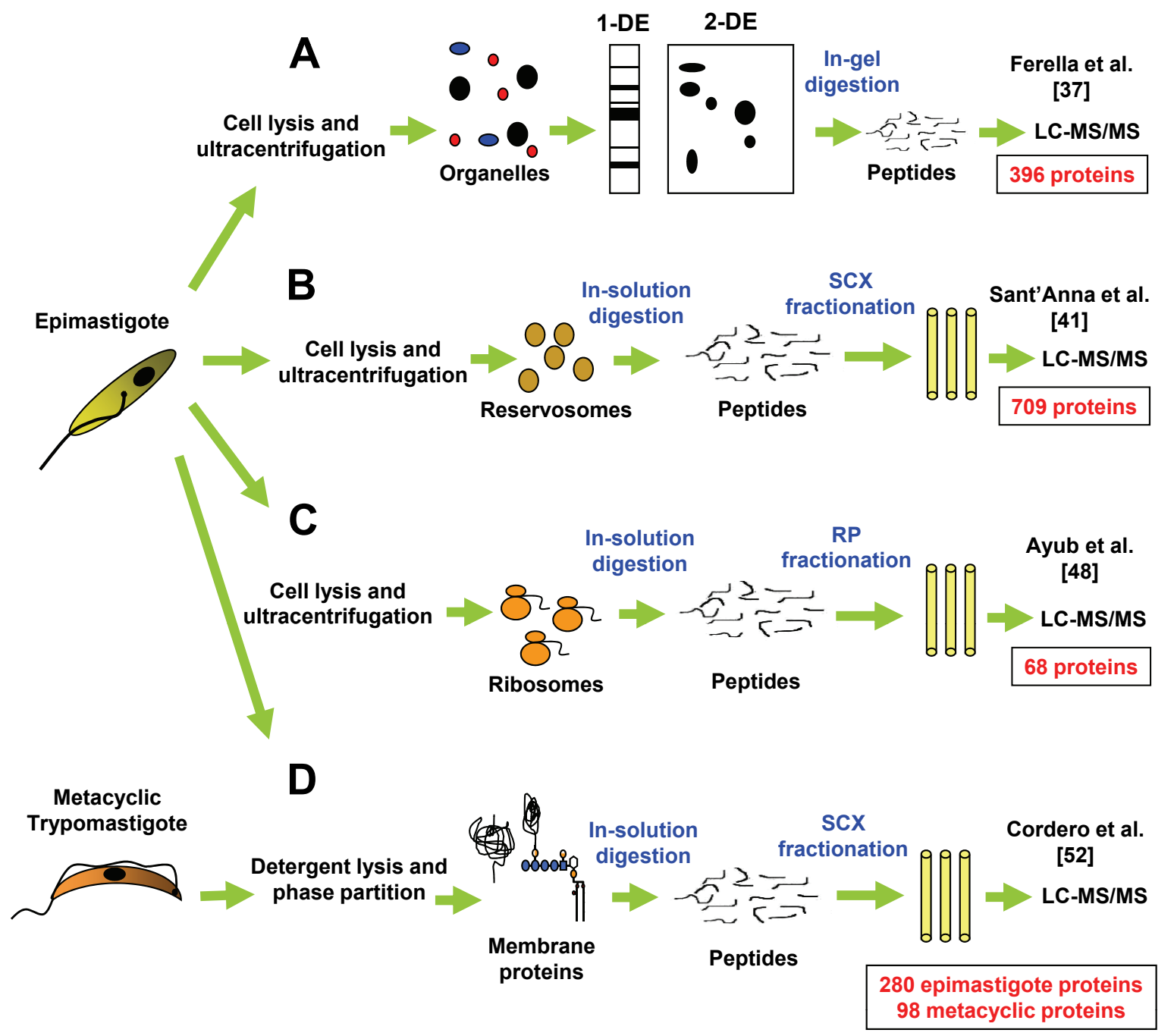

Fig. (2). Subcellular proteomic studies in T. cruzi. (A) Analysis of multi-organellar proteomics. Organelles were enriched by ultracentrifugation, and proteins were resolved by 1-DE or 2-DE, submitted to in-gel digestion with proteases and analyzed by LC-MS/MS. (B) Reservosomal proteomics. Reservosomes were purified by ultracentrifugation, and proteins submitted to in-solution digestion, fractionated by SCX, and analyzed by LC-MS/MS. (C) Ribosomal proteomics. 80S ribosomes were purified by differential ultracentrifugation, and proteins digested with trypsin, fractionated by RP chromatography, and analyzed by LC-MS/MS. (D) Membrane proteomics. Epimastigote and metacyclic trypomastigote membrane proteins were obtained by detergent extraction and phase partition. Proteins were then digestion in-solution, fractionated by SCX chromatography, and analyzed by LC-MS/MS.

proteins from insect developmental stages of $T$. cruzi (i.e., epimastigotes vs. metacyclic trypomastigotes), Cordero et al. [52] have recently carried out a detergent extraction followed by a phase partition. The phase partition is based on the property that, depending upon the temperature and concentration, some detergents aggregate, forming two immiscible phases, i.e., a detergent-poor and a detergent-rich phase [53]. Proteins recovered in the detergent-rich phase were digested with trypsin, fractionated by SCX chromatography and analyzed by LC-MS/MS (Fig. 2D). With this approach 98 metacyclic trypomastigote and 280 epimastigote proteins were identified [52]. The proteomic data clearly showed main differences between the noninfective/proliferative epimastigote stage and the infective/ nonproliferative metacyclic trypomastigote stage. For instance, on the one hand, metacyclic trypomastigotes showed to be highly enriched with surface glycosylphosphatidylinositol (GPI)-anchored glycoproteins, which are involved in host-cell invasion and evasion from the host immune response. On the other hand, epimastigotes showed to be enriched with proteins related to metabolic pathways, which support the fast proliferation of this parasite stage [52]. Interestingly, a large proportion $(\sim 50 \%)$ of the detergent-soluble epimastigote proteins was also identified in the reservosomal membrane fraction [41, 52].

\section{POSTTRANSLATIONAL MODIFICATIONS}

Currently, more the 300 PTMs are known to modify proteins in a physiological environment [26, 27]. PTMs have been shown to regulate many biological processes, such as, enzymatic activity, cellular localization of proteins, transport of molecules, binding to protein complexes, cell signaling, 
and interaction with other cells/organisms. Considering their importance, PTMs are still poorly studied in T. cruzi. Lately, comprehensive analyses of some PTMs have been providing new insights into key biological processes of $T$. cruzi and the role of parasite posttranslationally modified proteins in the pathogenesis Chagas disease. Large-scale analyses of histone modifications, protein phosphorylation, GPI-anchoring, glycosylation, and nitrosylation have been carried out, and the main findings of these studies are discussed below.

\section{Histone Modifications}

Histones are proteins that pack DNA into nucleosomes and play critical roles in the chromatin structure and epigenetic gene expression in higher eukaryotes [54, 55]. These proteins have high content of basic amino acid residues, which helps binding to the negative charge of nucleic acids. Among all known proteins, histones probably contain the largest number of PTMs, which include acetylation, methylation, phosphorylation, ubiquitination, SUMOylation, and ADP-ribosylation. These modifications play key roles controlling chromatin assembly and gene transcription rates [55, 56].

Unfortunately, T. cruzi histone sequences are divergent from those of higher eukaryotes, thus commercially available antibodies raised against specific modification sites may not properly recognize the parasite homologues. As a consequence, mapping PTMs of histones is a crucial step for studying epigenetics and histone roles in T. cruzi. However, the analysis of histone PTMs by MS-based proteomics is one of the most challenging tasks for the following reasons: (i) histones are rich in lysine and arginine residues; thus, trypsin, the most used enzyme to digest proteins in proteomic analysis, may provide too short peptides to be sequenced; (ii) a high number of possible PTMs and potential modification sites; and (iii) the difference between a trimethylated and an acetylated lysine residue is only $36 \mathrm{mDa}$, thus requiring very high-resolution MS analysis, which is not always readily available.

The first $T$. cruzi histone to be analyzed was the H1 [57]. The MS analysis of the intact protein suggested that it could be phosphorylated and had a series of methylations and/or acetylations. To study these potential PTMs in detail, purified histone $\mathrm{H} 1$ was digested with trypsin and analyzed by LC-MS/MS (Fig. 3A), resulting in the mapping of one acetylation at the $\mathrm{N}$-terminus and one phosphorylation at serine 12 [57]. Interestingly, this phosphorylation was enriched in nonproliferative/infective bloodstream trypomastigotes as compared to proliferative/non-infective epimastigotes [58]. This modification was also shown to be regulated according to the cell cycle possibly by a cyclin-dependent kinase-related enzyme (TzCRK3 or TzCRK1) [57]. Furthermore, antibodies raised specifically against the phosphorylated form of $\mathrm{H} 1$ enabled the study of its dynamics during the cell cycle [59].

The other $T$. cruzi histone to be analyzed was the H4 [60]. Using radiolabeled precursors, histone H4 was shown to be mainly acetylated and to a lesser extent, methylated. Since histone $\mathrm{H} 4$ has high content of lysine and arginine, it would generate too short peptides when treated with trypsin, thus da Cunha et al. [60] chose to digest the samples with either endoproteinase Glu-C or Arg-C. Resulting peptides were analyzed by MALDI-TOF/TOF-MS and LC-MS/MS and the resulting data suggested that histone $\mathrm{H} 4$ has many isoforms with different numbers of methylations and/or acetylations (Fig. 3A) [60]. To unambiguously distinguish between trimethylated and acetylated lysine residues, the samples were not only run in high mass accuracy instruments (i.e., MALDI-TOF/TOF-MS and ESI-QTOF-MS), but also the identification relied upon the presence of diagnostic immonium ions for each modification. With this approach, methylation at the $\mathrm{N}$-terminus, lysine acetylation at positions $4,10,14$, and 57, monomethylation at lysine 18, and dimethylation at arginine 53 were mapped [60]. By using antibodies raised against specific lysine acetylation positions, it was shown that histone modified at each position has a differential distribution in the nucleus [61]. Also, it was suggested that these modifications may play key roles in cell cycle, differentiation, and DNA repair mechanisms [61].

More recently, Respuela et al. [62] used antibodies raised against Tetrahymena acetylated histones, which have their lysine residues conversed as compared to the $T$. cruzi ones. These authors showed that acetylated and methylated histones are enriched in proximity to polycistronic clusters, suggesting that these modifications could be regulating transcription levels [62].

\section{Phosphorylation}

Protein phosphorylation is a ubiquitous PTM that regulates many cellular pathways. This modification occurs more frequently in the side chain of serine, threonine, and tyrosine residues in eukaryotes, but also can be found in histidine and aspartic acid residues mostly in prokaryotes. Although protein phosphorylation is perhaps the most investigated PTM, it is poorly explored in protozoan parasites and other pathogenic microorganisms. Since phosphorylation has already been targeted for the treatment of several illnesses, including central nervous system disorders and many types of cancer [63-65], it is predictable that it might be a very promising target for the development of chemotherapies against Chagas disease and other diseases caused by protozoan parasites [66, $67]$.

The $T$. cruzi kinome (the complete set of kinases) includes 190 genes that possibly code for kinases, of which $12 \%$ are atypical as compared to those found in higher eukaryotes $[67,68]$. On the other hand, T. cruzi has 86 phosphatase genes, being $40 \%$ atypical. Interestingly, the parasite lacks typical tyrosine kinase genes [69]. To identify the phosphoproteins and map the modified sites, Nakayasu et al. [70] performed a large-scale phosphoproteomic analysis of $T$. cruzi epimastigotes. The whole cell lysate was digested with trypsin, then phosphopeptides were enriched using immobilized metal-affinity chromatography (IMAC) and analyzed by LC-MS/MS (Fig. 3B). This analysis resulted in the identification of 220 phosphorylation sites (148 on serine, 57 on threonine, and 8 on tyrosine residues) in 119 distinct proteins [70]. Remarkably, approximately $4 \%$ of the phosphorylation sites were found in tyrosine residues, despite the absence of typical tyrosine kinases [70]. This proportion was similar to that found recently in $T$. brucei $[71,72]$. It has been proposed that kinases with dual specificity, such as Wee kinase, could be phosphorylating tyrosine residues 
$[67,68]$. However, experimental data is still necessary to confirm this hypothesis.

\section{Glycosylphosphatidylinositol (GPI) Anchoring}

Glycosylphosphatidylinositol (GPI) anchoring was first described in Trypanosoma brucei variant surface glycoprotein (VSG) and rat erythrocyte Thy-1 protein $[73,74]$. This is a ubiquitous posttranslational modification that attaches proteins into the cell surface and it is present in most known eukaryotes [75]. The basic structure of a GPI anchor consists of a lipid tail, which can be either a phosphatidylinositol (PI) or an inositolphosphorylceramide (IPC), and a glycan core, usually composed of three sequential mannose (Man) residues and a glucosamine (GlcN). The latter is linked to the myo-inositol residue of the lipid moiety $\left(\mathrm{Man}_{3}{ }^{-\mathrm{GlcN}}\right.$-PI or IPC). The protein is attached to the GPI through an ethanolamine phosphate (EtNP) group present in the third mannose distal from GlcN residue. The GPI anchor can be further modified with the addition of extra carbohydrate residues, EtNP, aminoethylphosphonate (AEP) on the glycan core, and fatty acid on the inositol ring [75]. GPIs can be also expressed in the protein-free forms, known as glycoinositolphospholipids (GIPLs), or linked to complex polysaccharide, as in the case of the Leishmania lipophosphoglycan (LPG) .

All protein sequences destined to receive a GPI-anchor have two conserved regions: i) a signal peptide at $\mathrm{N}$ terminus that drives the nascent protein into the ER; and ii) a hydrophobic C-terminal region known as the GPI-attachment signal peptide that docks the protein in the ER membrane. Once the protein is fully matured, the GPI-attachment signal peptide is cleaved and the GPI anchor is transferred en bloc to the newly formed C-terminus by an enzymatic complex called transamidase [76,77]. With the information of the Cterminal region and the amino acid residues surrounding the modification (omega, $\omega$ ) site, it is possible to predict potential GPI-anchored proteins with high accuracy [78]. For instance, it has been estimated that approximately $12 \%$ of the whole T. cruzi genome code for GPI-anchored proteins [79]. This number is much higher as compared to other related trypanosomatids, such as T. brucei [79] and Leishmania major [78], which have $1.5 \%$ and $1.0 \%$ of the protein sequences predicted to be GPI-anchored, respectively. In T. cruzi, the potential GPI-anchored sequences are concentrated into large multigene families (some of which with more than 1,000 genes) such as mucin-associated surface protein (MASP), mucin (comprising TcMUC I, TcMUC II, TcMUC III, TcSMUG S and TcSMUG L families), TS/gp85 glycoprotein, amastin, surface protease gp63, mucin-like protein, and TolT [79]. This large number of genes coding for putative GPI-anchored proteins could reflect in the expression of a highly complex surface coat of glycoconjugates, which could be explored by the parasite to effectively invade a vast array of cells and escape immune response within the mammalian host $[15,49]$, or to resist proteolytic enzymes within the insect-vector [79].

To identify the major surface GPI-anchored proteins on infective trypomastigote forms, Buscaglia et al. [80] performed a sequential solvent extraction and purification of these glycoconjugates by hydrophobic-interaction chromatography (HIC), followed by digestion with trypsin, and ESI-
MS/MS analysis. Using this approach, it was possible to sequence a short peptide attached to the GPI anchor and identify the protein sequence. The authors showed that the major surface GPI-anchored proteins of trypomastigotes belong to the highly diverse TcMUC II mucin family, which is encoded by hundreds of genes [14,80]. Interestingly, TcMUC II mucins and their isolated GPI anchors were previously shown to be strong proinflammatory molecules that activate Toll-like receptor 2 (TLR2)-mediated pathways, leading to production of cytokines (e.g., IL-12, TNF- $\alpha$ ), chemokines, and nitric oxide (NO) [15,81]. Other GPIanchored proteins, such as TS/gp85 and gp63 glycoproteins, were shown to be important during host-cell invasion and to protect the parasite against the host immune response. Furthermore, there are other major protein families predicted to be GPI-anchored (e.g., MASP, mucin-like, TolT) but with very little information about their expression and function $[14,15,82]$. Therefore, the in-depth analysis of major GPIanchored proteins will certainly provide new interesting insights into their relevance for the parasite biology and pathogenesis of Chagas disease.

Recent advances in technology made possible to analyze not only the most abundant GPI species but also to obtain a global view of the most comprehensive set of expressed GPIs, i.e., the GPIome. Until recently, one of the major hurdles for the analysis of the GPIome of T. cruzi was the dearth of a high-resolution chromatographic method for the purification of GPIs and GPI-anchored proteins (GPI-APs). Our hypothesis was that this setback was a result of the amphiphilic character of RP and HIC resins, which would interact with both hydrophilic (glycan) and hydrophobic (lipid) moieties of the GPI, thus considerably decreasing the resolving power of the separation. To solve this problem, we have introduced the use of C4-linked polystyrenedivinylbenzene (POROS R1) resin, which is exclusively hydrophobic and hence eliminates any eventual hydrophilic interactions. This methodology was first used for the global GPIomic analysis of epimastigote forms of T. cruzi [79]. Epimastigote-derived GIPLs and GPI-anchored proteins were extracted with organic solvents, and GPIs attached to proteins were further released by digestion with trypsin or proteinase $\mathrm{K}$, and analyzed by LC-MS/MS (Fig. 3C). Seventy-eight GIPL species were identified, of which 70 had not previously been described yet. Among the protein-linked GPIs, 12 distinct species were characterized, being 9 of them completely novel. Only one GPI-AP was sequenced and shown to belong to the TcSMUG S mucin family [79]. Interestingly, this family of mucins is highly glycosylated and short in length, with mature proteins predicted to have 56-85 amino acid residues. Also, TcSMUG S mucin sequences contain a central domain of threonine-rich repeats and very few trypsin sites located at the $\mathrm{N}$ - and C-termini, which would likely make these proteins somewhat resistant to trypsin and other proteolytic enzymes $[15,49]$. Together, the GPIomic data raises the possibility that the epimastigote highly diverse coat, rich in GIPLs and highly glycosylated, short GPI-APs could be involved in the protection of the parasite against digestive enzymes found in the reduviid midgut [79]. In addition, GIPLs were shown to be involved in the attachment of epimastigotes to the midgut epithelium of the insect vector [83]. 

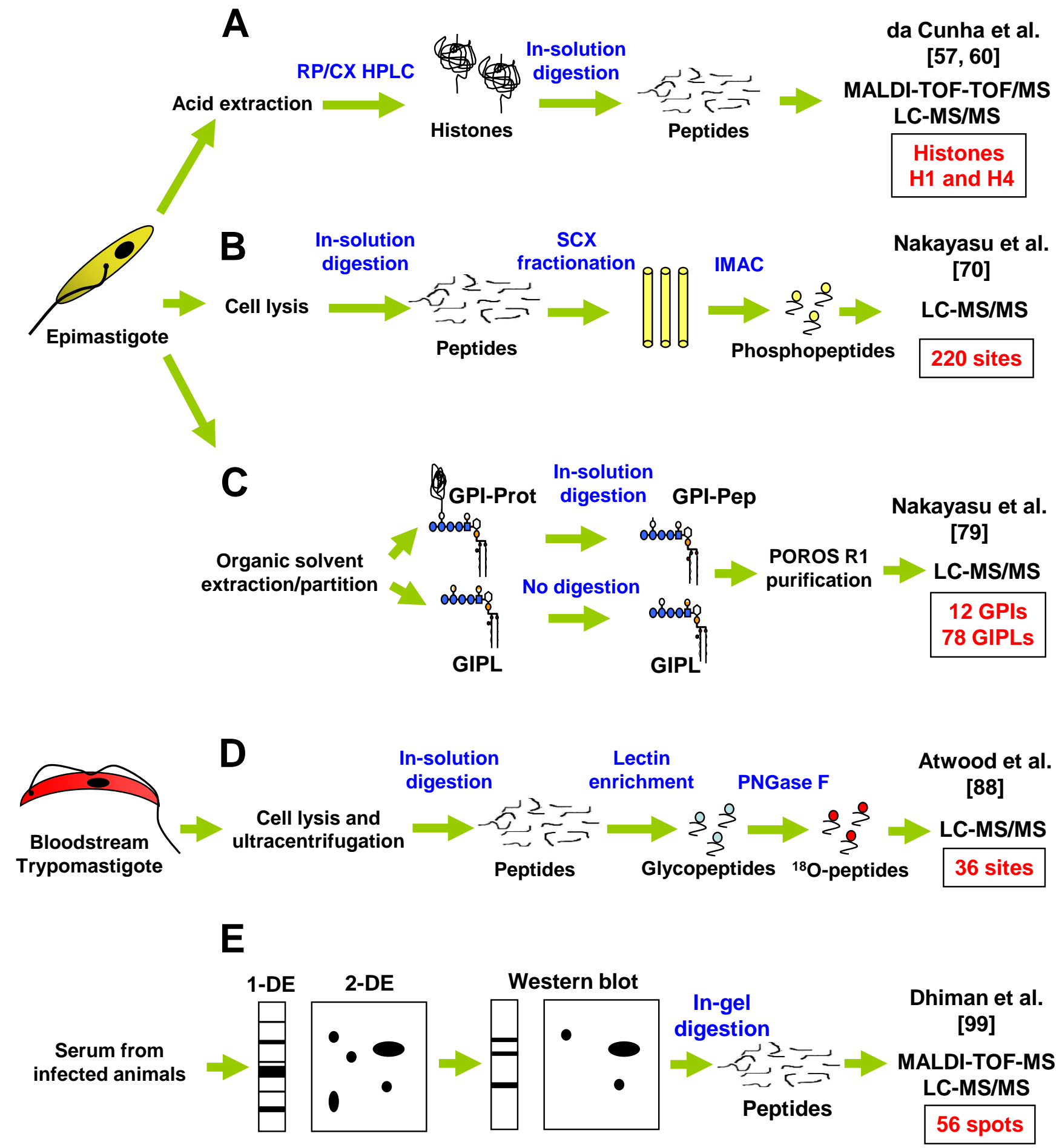

Fig. (3). Analysis of posttranslational modifications (PTMs) of T. cruzi proteins. (A) Histone PTMs. Histones were extracted with acid solutions, separated by RP or cation exchange (CX) high-performance liquid chromatography (HPLC), submitted to in-solution digestion, and analyzed by MALDI-TOF-TOF/MS or LC-MS/MS. (B) T. cruzi phosphoproteome. Whole cell lysate was digested with trypsin and fractionated by SCX chromatography. Phosphopeptides were enriched using immobilized metal-affinity chromatography (IMAC), and analyzed by LC-MS/MS. (C) T. cruzi GPIomics. Epimastigote GIPLs and GPI-anchored proteins (GPI-Prot) were extracted with organic solvents. GPIProt were digested with proteolytic enzymes, and both GPI-anchored peptides (GPI-Pep) and GIPLs were purified in POROS R1 columns, and analyzed by LC-MS/MS. (D) $N$-linked glycoproteome. Cells were lysed and separated into organellar and cytoplasmic/plasma membrane fractions. The proteins were digested and glycopeptides were enriched by lectin chromatography. Glycopeptides were treated with PNGase $\mathrm{F}$ in buffer prepared with ${ }^{18} \mathrm{O}$ water and analyzed by LC-MS/MS. (E) Analysis of nitrosylated serum proteins from $T$. cruzi-infected animals. Serum from infected animals were separated by 1-DE and 2-DE and submitted to Western blot using anti-3-nitrotyrosine antibodies. The reactive bands/spots were digested with proteases and analyzed by MALDI-MS or LC-MS/MS. 
Finally, the GPIomics technology opens new avenues for the large-scale investigation of GIPLs and GPI-APs not only from distinct $T$. cruzi stages, strains and lineages, but also from other medically relevant protozoan parasites, such as $T$. brucei, Plasmodium spp., Leishmania spp. and Toxoplasma gondii.

\section{Glycosylation}

$T$. cruzi has mainly 3 types of glycosylation: $N-, O-$, and phospho (P)-glycosylation $[84,85]$. Another common glycosylation type found in eukaryotes, the $\mathrm{O}$-linked $\mathrm{N}$ acetylglucosamine (or O-GlcNAcylation), was proposed to be absent in Trypanosoma spp., since these parasites lack the gene coding the $O$-linked $N$-acetylglucosaminyltransferase (OGT) enzyme, responsible for the transfer of $\beta$-GlcNAc to threonine or serine residue [86] . T. cruzi has 52 putative glycosyltransferase genes coding for enzymes that transfer nucleotide sugars. In addition, about 1,400 sequences of TS members are present in the genome [14]. This high number of glycosyltransferases is responsible for the synthesis of a variety of glycoconjugates, which are abundantly and differentially expressed in all T. cruzi stages. Many glycans and glycoconjugates are known to play key roles in the interaction of parasite with both insect and mammalian hosts [87].

Here, we will focus the discussion on protein glycosylation sites rather than the glycans themselves, since an excellent review was recently published about the latter topic [87]. In other to map $N$-linked glycosylation sites in trypomastigote proteins, Atwood et al. [88] used a sophisticated methodology. First, cells were lysed and separated into organellar and plasma membrane/cytosolic fractions. Both fractions were then submitted to trypsin digestion and glycopeptides were captured by lectin affinity chromatography using immobilized concanavalin A. The $N$-linked glycans were released with peptide- $N$-glycosidase F (PNGase F) in a buffer prepared with $\mathrm{H}_{2}{ }^{18} \mathrm{O}$ [88] (Fig. 3D). In this procedure, the modified asparagine residue is converted into aspartic acid, with the incorporation one ${ }^{18} \mathrm{O}$ atom, promoting a shift of 3 $\mathrm{Da}$ as compared to the unmodified asparagine residue [89]. This shift of $3 \mathrm{Da}$ is easily detected in the mass spectrometer, providing more confidence during the $\mathrm{N}$-glycosylated site mapping. With this approach, thirty-six glycosylation sites were mapped on 29 glycoproteins, including several TS, MASP, and dispersed gene family protein 1 (DGF-1) [88].

$O$-linked mucin-type glycosylation is abundantly found in surface GPI-anchored mucins $[15,49,90]$. $O$-glycosylation is particularly relevant for the elicitation of the protective (trypanolytic) humoral immune response conferred by anti$\alpha$-galactosyl (anti- $\alpha$-Gal) antibodies, found in high levels $(\sim 300 \mu \mathrm{g} / \mathrm{ml}$ serum$)$ in both acute and chronic stages of Chagas disease [91-94]. Although there are several reports and reviews $[15,49,90]$ regarding the structure of these $O$-linked glycans, there is no information about the modification sites, although they are predicted to be localized in threonine-rich regions.

Finally, P-glycosylation is another unique type of glycosylation reported to be present in $T$. cruzi. Phosphoglycans were initially isolated by mild acid hydrolysis of glycopeptides purified from a metacyclic trypomastigote lysate after pronase treatment followed by affinity chromatography using the monoclonal antibody WIC29.26, which is highly reactive to the Gp72 glycoprotein [85, 95]. These P-glycans were generally found linked to threonine and to a less extent, serine residues, through a Xyl-1- $\mathrm{PO}_{4}$ reducing terminus. The function of Gp72 remains elusive, but this glycoprotein seems to be involved in complement activation, maintenance of parasite morphology, and flagellar adhesion [96]. More recently, P-glycosylation was also found in oligomannosides of the NETNES glycoconjugate, a GPI-anchored glycopeptide containing two P-glycans linked to serine and threonine residues through a Man-1- $\mathrm{PO}_{4}$ reducing terminus [97]. The biological function of NETNES, however, remains to be determined.

\section{Protein Nitrosylation}

Oxidative stress is part of a proinflammatory response against $T$. cruzi infection [98]. During this process, reactive oxygen and nitrogen species are produced and may cause protein carbonylation and nitrosylation, respectively. During an infection, inducible nitric oxide synthase (iNOS) is overexpressed, resulting in the production high amounts of NO. $\mathrm{NO}$ in the presence of oxygen radicals is converted into peroxynitrite, which nitrosylates tyrosine and cysteine residues [98]. Recently, Dhiman et al. [99] investigated the presence of 3-nitrotyrosine (3NT) in proteins from plasma of acutely and chronically infected mice. Plasma from these animals were depleted from serum albumin, separated in 1-DE or 2DE and submitted to Western blot assay using anti-3NT antibodies (Fig. 3E). Fifty-six positive spots for 3NT were analyzed by MALDI-MS and LC-MS/MS, leading to the identification of 50 of them, including $\operatorname{IgG}, \operatorname{IgA}$, serum albumin, fibrinogen, and apolipoprotein A-I [99]. Furthermore, protein-3NT was recently shown to be a reliable indicator of inflammation in patients with Chagas disease [100]. Whether 3NT-modified proteins have their function(s) somehow affected is a question that still needs to be addressed.

\section{CONCLUDING REMARKS}

Advances in proteomic technologies have been having a great impact in the fields of biology and biomedical sciences. The application of this technology to $T$. cruzi-derived samples has been helping to build not only a catalog of expressed proteins in different parasite stages but also allowing the characterization of proteins from specific organelles and/or with different PTMs. The analysis of subcellular proteomics and PTMs combined with biochemical, immunological and cell biology approaches is accelerating the discovery of new biological functions of a considerable number of $T$. cruzi molecules. Still, the major challenge remains the preparation of highly enriched organelles and fractions of posttranslationally modified proteins for proteomic analysis. We believe the in-depth proteomic studies of distinct stages, lineages, and strains of $T$. cruzi will further the development of new, more effective therapeutic interventions against this deadly parasite.

\section{ABBREVIATIONS}

$\begin{array}{ll}\text { 1-DE } & =\text { one-dimensional gel electrophoresis } \\ 2-\mathrm{DE} & =\text { two-dimensional gel electrophoresis } \\ \text { AEP } & =\text { aminoethylphosphonate }\end{array}$




$$
\begin{aligned}
& \text { cryo-EM } \quad=\text { cryo-electron microscopy } \\
& \text { DGF-1 = dispersed gene family protein } 1 \\
& \text { ER } \quad=\text { endoplasmic reticulum } \\
& \text { ESI-MS/MS = electrospray ionization-tandem mass } \\
& \text { spectrometry } \\
& \text { ESI-QTOF-MS = electrospray ionization quadrupole- } \\
& \text { time-of-flight mass spectrometry } \\
& \text { EtNP = ethanolaminephosphate } \\
& \text { GIPL }=\text { glycoinositolphospholipid } \\
& \text { GPI = glycosylphosphatidylinositol } \\
& \text { HILIC = hydrophilic-interaction chromatogra- } \\
& \text { phy } \\
& \text { IMAC }=\text { immobilized metal-affinity chromato- } \\
& \text { graphy } \\
& \text { iNOS }=\text { inducible nitric oxide synthase } \\
& \text { LC-MS/MS = liquid chromatography-tandem mass } \\
& \text { spectrometry } \\
& \text { LPG } \quad=\text { lipophosphoglycan } \\
& \text { MALDI-MS }=\text { matrix-assisted laser desorption/ioniza- } \\
& \text { tion-mass spectrometry } \\
& \text { MALDI-TOF/ = matrix-assisted laser desorption/ioniza- } \\
& \text { TOF-MS tion-tandem time-of-flight mass spec- } \\
& \text { trometry } \\
& \text { MASP }=\text { mucin-associated surface protein } \\
& \text { nanoHPLC or }=\text { nanoflow high-performance liquid } \\
& \text { nanoLC chromatography } \\
& \mathrm{NO} \quad=\text { nitric oxide } \\
& \text { PMF } \quad=\text { peptide mass fingerprint } \\
& \text { PNGase F }=\text { peptide- } N \text {-glycosidase } \mathrm{F} \\
& \text { PTM }=\text { protein posttranslational modification } \\
& \mathrm{RP} \quad=\text { reverse phase } \\
& \text { RHS } \quad=\text { retrotransposon hot spot } \\
& \text { SAX }=\text { strong-anion exchange } \\
& \text { SCX }=\text { strong cation-exchange } \\
& \text { SL } \quad=\text { spliced leader } \\
& \text { TLR2 }=\text { Toll-like receptor } 2 \\
& \text { TLR6 }=\text { Toll-like receptor } 6 \\
& \text { TS }=\text { trans-sialidase }
\end{aligned}
$$

\section{ACKNOWLEDGEMENTS}

This study was funded by the grants 1R01AI070655, 2S06GM00812, 5G12RR008124-16A1, and 5G12RR00 8124-16A1S1 from the National Institutes of Health (NIH). We thank the Biomolecule Analysis Core Facility at the Border Biomedical Research Center (BBRC), UTEP, funded by NIH grants 5G12RR008124-16A1 and 5G12RR00812416A1S1 for the full access to the LC-MS instrumentation used in our studies. E.S.N. was partially supported by the George A. Krutilek memorial graduate scholarship from Graduate School, UTEP.

\section{REFERENCES}

[1] Stuart K, Brun R, Croft S, et al. Kinetoplastids: related protozoan pathogens, different diseases. J Clin Invest 2008; 118: 1301-10.

[2] Dias JC, Silveira AC, Schofield CJ. The impact of Chagas disease control in Latin America: a review. Mem Inst Oswaldo Cruz 2002; 97: 603-12.

[3] Tarleton RL, Reithinger R, Urbina JA, Kitron U, Gurtler RE. The challenges of Chagas Disease- grim outlook or glimmer of hope. PLoS Med 2007;4:e332.

[4] Barrett MP, Burchmore RJ, Stich A, et al. The trypanosomiases. Lancet 2003; 362: 1469-80.

[5] Bern C, Montgomery SP, Herwaldt BL, et al. Evaluation and treatment of chagas disease in the United States: a systematic review. JAMA 2007; 298: 2171-81.

[6] Piron M, Verges M, Munoz J, et al. Seroprevalence of Trypanosoma cruzi infection in at-risk blood donors in Catalonia (Spain). Transfusion 2008; 48: 1862-8.

[7] Schmunis GA, Yadon ZE. Chagas disease: A Latin American health problem becoming a world health problem. Acta Trop 2010; 115(1-2): 14-21.

[8] Tyler KM, Engman DM. The life cycle of Trypanosoma cruzi revisited. Int J Parasitol 2001; 31: 472-81.

[9] Pereira KS, Schmidt FL, Guaraldo AM, Franco RM, Dias VL, Passos LA. Chagas' disease as a foodborne illness. J Food Prot 2009; 72: 441-6.

[10] Urbina JA. Specific chemotherapy of Chagas disease: Relevance, current limitations and new approaches. Acta Trop 2010; 115(1-2): 55-68.

[11] Garg N, Bhatia V. Current status and future prospects for a vaccine against American trypanosomiasis. Expert Rev Vaccines 2005; 4: 867-80.

[12] Cazorla SI, Frank FM, Malchiodi EL. Vaccination approaches against Trypanosoma cruzi infection. Expert Rev Vaccines 2009; 8: 921-35.

[13] Tarleton RL. New approaches in vaccine development for parasitic infections. Cell Microbiol 2005; 7: 1379-86.

[14] El-Sayed NM, Myler PJ, Bartholomeu DC, et al. The genome sequence of Trypanosoma cruzi, etiologic agent of Chagas disease. Science 2005; 309: 409-15.

[15] Acosta-Serrano A, Hutchinson C, Nakayasu ES, Almeida IC, Carrington $\mathrm{M}$. Comparison and evolution of the surface architecture of trypanosomatid parasites. In: D. Barry JM, R. McCulloch, and A. Acosta-Serrano, Eds. Trypanosomes - After the Genome. Norwich, UK: Horizon Scientific Press 2007.

[16] Pena SD, Machado CR, Macedo AM. Trypanosoma cruzi: ancestral genomes and population structure. Mem Inst Oswaldo Cruz 2009; 104(Suppl 1): 108-14.

[17] Buscaglia CA, Di Noia JM. Trypanosoma cruzi clonal diversity and the epidemiology of Chagas' disease. Microbes Infect 2003; 5 : 419-27.

[18] Zingales B, Andrade SG, Briones MR, et al. A new consensus for Trypanosoma cruzi intraspecific nomenclature: second revision meeting recommends TcI to TcVI. Mem Inst Oswaldo Cruz 2009; 104: 1051-4.

[19] Jager AV, De Gaudenzi JG, Cassola A, D'Orso I, Frasch AC. mRNA maturation by two-step trans-splicing/polyadenylation processing in trypanosomes. Proc Natl Acad Sci USA 2007; 104: 2035-42.

[20] Aebersold R, Mann M. Mass spectrometry-based proteomics. Nature 2003; 422: 198-207.

[21] Pandey A, Mann M. Proteomics to study genes and genomes. Nature 2000; 405: 837-46.

[22] Yates JR, 3rd. Mass spectrometry and the age of the proteome. J Mass Spectrom 1998; 33: 1-19.

[23] Motoyama A, Yates JR, 3rd. Multidimensional LC separations in shotgun proteomics. Anal Chem 2008; 80: 7187-93.

[24] Jensen ON. Modification-specific proteomics: characterization of post-translational modifications by mass spectrometry. Curr Opin Chem Biol 2004; 8: 33-41.

[25] Mann M, Jensen ON. Proteomic analysis of post-translational modifications. Nat Biotechnol 2003; 21: 255-61.

[26] Witze ES, Old WM, Resing KA, Ahn NG. Mapping protein posttranslational modifications with mass spectrometry. Nat Methods 2007; 4: 798-806. 
[27] Zhao Y, Lee WN, Xiao GG. Quantitative proteomics and biomarker discovery in human cancer. Expert Rev Proteomics 2009; 6: 115-8.

[28] Atwood JA, Weatherly DB, Minning TA, et al. The Trypanosoma cruzi proteome. Science 2005; 309: 473-6.

[29] Magalhaes AD, Charneau S, Paba J, et al. Trypanosoma cruzi alkaline 2-DE: Optimization and application to comparative proteome analysis of flagellate life stages. Proteome Sci 2008; 6: 24.

[30] Paba J, Ricart CA, Fontes W, et al. Proteomic analysis of Trypanosoma cruzi developmental stages using isotope-coded affinity tag reagents. J Proteome Res 2004; 3: 517-24.

[31] Paba J, Santana JM, Teixeira AR, Fontes W, Sousa MV, Ricart CA. Proteomic analysis of the human pathogen Trypanosoma cruzi. Proteomics 2004; 4: 1052-9.

[32] Parodi-Talice A, Duran R, Arrambide N, et al. Proteome analysis of the causative agent of Chagas disease: Trypanosoma cruzi. Int J Parasitol 2004; 34: 881-6.

[33] Parodi-Talice A, Monteiro-Goes V, Arrambide N, et al. Proteomic analysis of metacyclic trypomastigotes undergoing Trypanosoma cruzi metacyclogenesis. J Mass Spectrom 2007; 42: 1422-32.

[34] Andrade HM, Murta SM, Chapeaurouge A, Perales J, Nirde P, Romanha AJ. Proteomic analysis of Trypanosoma cruzi resistance to Benznidazole. J Proteome Res 2008; 7: 2357-67.

[35] De Souza W. Basic cell biology of Trypanosoma cruzi. Curr Pharm Des 2002; 8: 269-85.

[36] de Souza W. Structural organization of Trypanosoma cruzi. Mem Inst Oswaldo Cruz 2009; 104 Suppl 1: 89-100.

[37] Ferella M, Nilsson D, Darban H, et al. Proteomics in Trypanosoma cruzi--localization of novel proteins to various organelles. Proteomics 2008; 8: 2735-49.

[38] Cunha-e-Silva N, Sant'Anna C, Pereira MG, Porto-Carreiro I, Jeovanio AL, de Souza W. Reservosomes: multipurpose organelles? Parasitol Res 2006; 99: 325-7.

[39] de Souza W, Sant'Anna C, Cunha-e-Silva NL. Electron microscopy and cytochemistry analysis of the endocytic pathway of pathogenic protozoa. Prog Histochem Cytochem 2009; 44: 67-124.

[40] Sant'Anna C, Parussini F, Lourenco D, de Souza W, Cazzulo JJ, Cunha-e-Silva NL. All Trypanosoma cruzi developmental forms present lysosome-related organelles. Histochem Cell Biol 2008; 130: 1187-98.

[41] Sant'Anna C, Nakayasu ES, Pereira MG, et al. Subcellular proteomics of Trypanosoma cruzi reservosomes. Proteomics 2009; 9: 1782-94.

[42] Broadhead R, Dawe HR, Farr H, et al. Flagellar motility is required for the viability of the bloodstream trypanosome. Nature 2006; 440: 224-7.

[43] Colasante C, Ellis M, Ruppert T, Voncken F. Comparative proteomics of glycosomes from bloodstream form and procyclic culture form Trypanosoma brucei brucei. Proteomics 2006; 6: 327593.

[44] Panigrahi AK, Ogata Y, Zikova A, et al. A comprehensive analysis of Trypanosoma brucei mitochondrial proteome. Proteomics 2009; 9: 434-50.

[45] Martinez-Calvillo S, Vizuet-de-Rueda JC, Florencio-Martinez LE, Manning-Cela RG, Figueroa-Angulo EE. Gene expression in trypanosomatid parasites. J Biomed Biotechnol 2010; 2010: 525241.

[46] Lukes J, Hashimi H, Zikova A. Unexplained complexity of the mitochondrial genome and transcriptome in kinetoplastid flagellates. Curr Genet 2005; 48: 277-99.

[47] Gao H, Ayub MJ, Levin MJ, Frank J. The structure of the $80 \mathrm{~S}$ ribosome from Trypanosoma cruzi reveals unique rRNA components. Proc Natl Acad Sci USA 2005; 102: 10206-11.

[48] Ayub MJ, Atwood J, Nuccio A, Tarleton R, Levin MJ. Proteomic analysis of the Trypanosoma cruzi ribosomal proteins. Biochem Biophys Res Commun 2009; 382: 30-4.

[49] Buscaglia CA, Campo VA, Frasch AC, Di Noia JM. Trypanosoma cruzi surface mucins: host-dependent coat diversity. Nat Rev Microbiol 2006; 4: 229-36.

[50] Lu B, McClatchy DB, Kim JY, Yates JR, 3rd. Strategies for shotgun identification of integral membrane proteins by tandem mass spectrometry. Proteomics 2008; 8: 3947-55.

[51] Wu CC, Yates JR, 3rd. The application of mass spectrometry to membrane proteomics. Nat Biotechnol 2003; 21: 262-7.

[52] Cordero EM, Nakayasu ES, Gentil LG, Yoshida N, Almeida IC, da Silveira JF. Proteomic analysis of detergent-solubilized membrane proteins from insect-developmental forms of Trypanosoma cruzi. $\mathbf{J}$ Proteome Res 2009; 8: 3642-52.

[53] Ko YG, Thompson GA, Jr. Purification of glycosylphosphatidylinositol-anchored proteins by modified triton X-114 partitioning and preparative gel electrophoresis. Anal Biochem 1995; 224: 16672 .

[54] Bernstein BE, Meissner A, Lander ES. The mammalian epigenome. Cell 2007;128:669-81.

[55] Latham JA, Dent SY. Cross-regulation of histone modifications. Nat Struct Mol Biol 2007; 14: 1017-24.

[56] Siuti N, Kelleher NL. Decoding protein modifications using topdown mass spectrometry. Nat Methods 2007; 4: 817-21.

[57] Da Cunha JP, Nakayasu ES, Elias MC, et al. Trypanosoma cruzi histone $\mathrm{H} 1$ is phosphorylated in a typical cyclin dependent kinase site accordingly to the cell cycle. Mol Biochem Parasitol 2005; 140: $75-86$.

[58] Marques Porto R, Amino R, Elias MC, Faria M, Schenkman S. Histone $\mathrm{H} 1$ is phosphorylated in non-replicating and infective forms of Trypanosoma cruzi. Mol Biochem Parasitol 2002; 119: 265-71.

[59] Gutiyama LM, da Cunha JP, Schenkman S. Histone H1 of Trypanosoma cruzi is concentrated in the nucleolus region and disperses upon phosphorylation during progression to mitosis. Eukaryot Cell 2008; 7 : 560-8.

[60] da Cunha JP, Nakayasu ES, de Almeida IC, Schenkman S. Posttranslational modifications of Trypanosoma cruzi histone H4. Mol Biochem Parasitol 2006; 150: 268-77.

[61] Nardelli SC, da Cunha JP, Motta MC, Schenkman S. Distinct acetylation of Trypanosoma cruzi histone $\mathrm{H} 4$ during cell cycle, parasite differentiation, and after DNA damage. Chromosoma 2009; 118: 487-99.

[62] Respuela P, Ferella M, Rada-Iglesias A, Aslund L. Histone acetylation and methylation at sites initiating divergent polycistronic transcription in Trypanosoma cruzi. J Biol Chem 2008; 283: 15884-92.

[63] Chico LK, Van Eldik LJ, Watterson DM. Targeting protein kinases in central nervous system disorders. Nat Rev Drug Discov 2009; 8: 892-909.

[64] Kim LC, Song L, Haura EB. Src kinases as therapeutic targets for cancer. Nat Rev Clin Oncol 2009; 6: 587-95.

[65] Zhang J, Yang PL, Gray NS. Targeting cancer with small molecule kinase inhibitors. Nat Rev Cancer 2009; 9: 28-39.

[66] de Azevedo WF, Jr., Soares MB. Selection of targets for drug development against protozoan parasites. Curr Drug Targets 2009; 10: $193-201$.

[67] Naula C, Parsons M, Mottram JC. Protein kinases as drug targets in trypanosomes and Leishmania. Biochim Biophys Acta 2005; 1754: 151-9.

[68] Parsons M, Worthey EA, Ward PN, Mottram JC. Comparative analysis of the kinomes of three pathogenic trypanosomatids: Leishmania major, Trypanosoma brucei and Trypanosoma cruzi. BMC Genomics 2005; 6: 127.

[69] Brenchley R, Tariq H, McElhinney H, et al. The TriTryp phosphatome: analysis of the protein phosphatase catalytic domains. BMC Genomics 2007; 8: 434.

[70] Nakayasu ES, Gaynor MR, Sobreira TJ, Ross JA, Almeida IC. Phosphoproteomic analysis of the human pathogen Trypanosoma cruzi at the epimastigote stage. Proteomics 2009; 9: 3489-506.

[71] Nett IR, Davidson L, Lamont D, Ferguson MA. Identification and specific localization of tyrosine-phosphorylated proteins in Trypanosoma brucei. Eukaryot Cell 2009; 8: 617-26.

[72] Nett IR, Martin DM, Miranda-Saavedra D, et al. The phosphoproteome of bloodstream form Trypanosoma brucei, causative agent of African sleeping sickness. Mol Cell Proteomics 2009; 8: 1527-38.

[73] Homans SW, Ferguson MA, Dwek RA, Rademacher TW, Anand R, Williams AF. Complete structure of the glycosyl phosphatidylinositol membrane anchor of rat brain Thy-1 glycoprotein. Nature 1988; 333: 269-72.

[74] Ferguson MA, Homans SW, Dwek RA, Rademacher TW. Glycosyl-phosphatidylinositol moiety that anchors Trypanosoma brucei variant surface glycoprotein to the membrane. Science 1988; 239: 753-9.

[75] McConville MJ, Ferguson MA. The structure, biosynthesis and function of glycosylated phosphatidylinositols in the parasitic protozoa and higher eukaryotes. Biochem J 1993; 294 ( Pt 2): 30524. 
[76] Kinoshita T, Fujita M, Maeda Y. Biosynthesis, remodeling and functions of mammalian GPI-anchored proteins: recent progress. J Biochem 2008; 144(3): 287-94.

[77] Orlean P, Menon AK. Thematic review series: lipid posttranslational modifications. GPI anchoring of protein in yeast and mammalian cells, or: how we learned to stop worrying and love glycophospholipids. J Lipid Res 2007; 48: 993-1011.

[78] Poisson G, Chauve C, Chen X, Bergeron A. FragAnchor: a largescale predictor of glycosylphosphatidylinositol anchors in eukaryote protein sequences by qualitative scoring. Genomics Proteomics Bioinformatics 2007; 5: 121-30.

[79] Nakayasu ES, Yashunsky DV, Nohara LL, Torrecilhas AC, Nikolaev AV, Almeida IC. GPIomics: global analysis of glycosylphosphatidylinositol-anchored molecules of Trypanosoma cruzi. Mol Syst Biol 2009; 5: 261.

[80] Buscaglia CA, Campo VA, Di Noia JM, et al. The surface coat of the mammal-dwelling infective trypomastigote stage of Trypanosoma cruzi is formed by highly diverse immunogenic mucins. J Biol Chem 2004; 279: 15860-9.

[81] Almeida IC, Gazzinelli RT. Proinflammatory activity of glycosylphosphatidylinositol anchors derived from Trypanosoma cruzi: structural and functional analyses. J Leukoc Biol 2001; 70: 467-77.

[82] Bartholomeu DC, Cerqueira GC, Leao AC, et al. Genomic organization and expression profile of the mucin-associated surface protein (masp) family of the human pathogen Trypanosoma cruzi. Nucleic Acids Res 2009; 37: 3407-17.

[83] Nogueira NF, Gonzalez MS, Gomes JE, et al. Trypanosoma cruzi: involvement of glycoinositolphospholipids in the attachment to the luminal midgut surface of Rhodnius prolixus. Exp Parasitol 2007; 116: $120-8$.

[84] McConville MJ, Mullin KA, Ilgoutz SC, Teasdale RD. Secretory pathway of trypanosomatid parasites. Microbiol Mol Biol Rev 2002; 66: 122-54.

[85] Haynes PA. Phosphoglycosylation: a new structural class of glycosylation? Glycobiology 1998; 8: 1-5.

[86] Banerjee S, Robbins PW, Samuelson J. Molecular characterization of nucleocytosolic O-GlcNAc transferases of Giardia lamblia and Cryptosporidium parvum. Glycobiology 2009; 19: 331-6.

[87] de Lederkremer RM, Agusti R. Glycobiology of Trypanosoma cruzi. Adv Carbohydr Chem Biochem 2009; 62: 311-66.

[88] Atwood JA, Minning T, Ludolf F, et al. Glycoproteomics of Trypanosoma cruzi trypomastigotes using subcellular fractionation, lectin affinity, and stable isotope labeling. J Proteome Res 2006; 5: 3376-84.
[89] Kaji H, Saito H, Yamauchi Y, et al. Lectin affinity capture, isotope-coded tagging and mass spectrometry to identify $\mathrm{N}$-linked glycoproteins. Nat Biotechnol 2003; 21: 667-72.

[90] Acosta-Serrano A, Almeida IC, Freitas-Junior LH, Yoshida N, Schenkman S. The mucin-like glycoprotein super-family of Trypanosoma cruzi: structure and biological roles. Mol Biochem Parasitol 2001; 114: 143-50.

[91] Almeida IC, Ferguson MA, Schenkman S, Travassos LR. GPIanchored glycoconjugates from Trypanosoma cruzi trypomastigotes are recognized by lytic anti-alpha-galactosyl antibodies isolated from patients with chronic Chagas' disease. Braz J Med Biol Res 1994; 27: 443-7.

[92] Gazzinelli RT, Pereira ME, Romanha A, Gazzinelli G, Brener Z. Direct lysis of Trypanosoma cruzi: a novel effector mechanism of protection mediated by human anti-gal antibodies. Parasite Immunol 1991; 13: 345-56.

[93] Almeida IC, Milani SR, Gorin PA, Travassos LR. Complementmediated lysis of Trypanosoma cruzi trypomastigotes by human anti-alpha-galactosyl antibodies. J Immunol 1991; 146: 2394-400.

[94] Pereira-Chioccola VL, Acosta-Serrano A, Correia de Almeida I, et al. Mucin-like molecules form a negatively charged coat that protects Trypanosoma cruzi trypomastigotes from killing by human anti-alpha-galactosyl antibodies. J Cell Sci 2000; 113 ( Pt 7): 1299307.

[95] Haynes PA, Ferguson MA, Cross GA. Structural characterization of novel oligosaccharides of cell-surface glycoproteins of Trypanosoma cruzi. Glycobiology 1996; 6: 869-78.

[96] Haynes PA, Russell DG, Cross GA. Subcellular localization of Trypanosoma cruzi glycoprotein Gp72. J Cell Sci 1996; 109 ( Pt 13): 2979-88.

[97] Macrae JI, Acosta-Serrano A, Morrice NA, Mehlert A, Ferguson MA. Structural characterization of NETNES, a novel glycoconjugate in Trypanosoma cruzi epimastigotes. J Biol Chem 2005; 280: 12201-11.

[98] Gupta S, Wen JJ, Garg NJ. Oxidative stress in chagas disease. Interdiscip Perspect Infect Dis 2009; 2009: 190354.

[99] Dhiman M, Nakayasu ES, Madaiah YH, et al. Enhanced nitrosative stress during Trypanosoma cruzi infection causes nitrotyrosine modification of host proteins: implications in Chagas' disease. Am J Pathol 2008; 173: 728-40.

[100] Dhiman M, Estrada-Franco JG, Pando JM, et al. Increased myeloperoxidase activity and protein nitration are indicators of inflammation in patients with Chagas' disease. Clin Vaccine Immunol 2009; 16: 660-6.

(C) Almeida and Nakayasu; Licensee Bentham Open.

This is an open access article licensed under the terms of the Creative Commons Attribution Non-Commercial License (http://creativecommons.org/licenses/by-nc/3.0/) which permits unrestricted, non-commercial use, distribution and reproduction in any medium, provided the work is properly cited. 